\title{
Invasive ductal carcinoma within borderline phyllodes tumor with lymph node metastases: A case report and review of the literature
}

\author{
DI WU ${ }^{1}$, HAIPENG ZHANG $^{2}$, LIANG GUO ${ }^{3}$, XU YAN $^{3}$ and ZHIMIN FAN ${ }^{1}$ \\ Departments of ${ }^{1}$ Breast Surgery, ${ }^{2}$ Obstetrics and ${ }^{3}$ Pathology, First Hospital of Jilin University, \\ Changchun, Jilin 130021, P.R. China
}

Received October 15, 2014; Accepted July 30, 2015

DOI: $10.3892 / \mathrm{ol} .2016 .4238$

\begin{abstract}
Phyllodes tumor (PT) is a rare type of biphasic fibroepithelial neoplasm that may coexist with a breast tumor in rare cases. In the current study, a 52-year-old female presented with a left breast lump. Mammography and sonographic examination results suggested a diagnosis of malignant tumor. Histological analysis revealed a borderline PT with invasive ductal carcinoma (IDC) within the tumor. Due to the presence of a single micrometastasis in three of the sentinel lymph nodes, the patient underwent modified radical mastectomy. The excised tumor contained triple negative breast cancer; therefore, postoperative treatment included six cycles of chemotherapy and 25 cycles of radiotherapy. The patient exhibited no recurrence and no metastatic disease at the 23-month follow-up examination. Thus, the present study discussed the case of a female patient that presented with IDC within borderline PT and reviewed the literature on this rare type of neoplasm. Various types of breast carcinoma have been identified to coexist with PT in different masses; however, no standard therapeutic regimen has been established for the coexistence of PT and breast cancer in the same mass. The present study indicates that determination of an appropriate treatment strategy predominantly depends on the characteristics of the individual breast tumor.
\end{abstract}

\section{Introduction}

Phyllodes tumor (PT) is a rare type of biphasic fibroepithelial neoplasm that accounts for $<1 \%$ of all breast tumors and represents $2-3 \%$ of fibroepithelial neoplasms $(1,2)$ with a peak age of incidence of 45-49 years $(3,4)$. According to the standards set by the World Health Organization (WHO), PTs may be classified as benign, borderline or malignant based on the degree of stromal cell atypia, mitotic status, degree of stromal overgrowth,

Correspondence to: Dr Zhimin Fan, Department of Breast Surgery, First Hospital of Jilin University, 71 Xinmin Street, Changchun, Jinlin 130021, P.R. China

E-mail: fanzhimn@163.com

Key words: phyllodes tumor, breast cancer, coexist, diagnosis, treatment tumor necrosis and appearance of tumor margins (5). PTs are predominantly benign with only $\sim 10 \%$ identified as malignant. The majority of malignant transformation of PTs typically occurs in the stromal compartment and rarely in the epithelial compartment. Breast carcinoma within PT accounts for 1-2\% of all PTs (6). Surgery is considered the standard treatment for PT (7). Invasive ductal carcinomas (IDC) of the breast accounts for $80 \%$ of all breast cancers, and these tumors demonstrate a worse survival rate than invasive lobular carcinoma (8), with overall 5-year survival rates of 84.1 and $85.6 \%$, respectively (9). An IDC that is incidentally found within a borderline PT has been reported only once before in the literature (10). The current study presents a case of IDC within a borderline PT, and reviews 32 cases of breast carcinoma within a PT that have been reported in the literature.

\section{Case report}

In July 2012, a 52-year-old female presented to the Department of Breast Surgery, First Hospital of Jilin University (Changchun, China) with a firm, palpable, irregularly-shaped lump with an ill-defined margin in the outer upper quadrant of the left breast. The lump, which was originally identified by the patient 6 months previously, had increased in size from $1.5 \times 1.0 \mathrm{~cm}$ at presentation to $2.5 \times 2.0 \mathrm{~cm}$ after 3 months. Physical examination revealed that the tumor did not adhere to or invade the overlying skin or the thoracic wall. Enlarged axillary lymph nodes were not identified upon physical examination. Mammography imaging revealed a high-density mass with a diameter of $2.5 \mathrm{~cm}$ and an irregular margin (Fig. 1), and sonographic examination demonstrated a partially ill-defined hypoechoic mass with a diameter of $2.1 \mathrm{~cm}$ (Fig. 2). A core needle biopsy revealed borderline or malignant PT with a breast carcinoma component.

The diagnosis was determined by analysis of the core needle biopsy, as follows. The tumor was well-circumscribed and $3.0 \times 2.5 \times 1.2 \mathrm{~cm}$ in size, according to macroscopic examination. The mitotic count in the most active area was 2-4 mitoses per 10 high-powered fields. Based on an increase in the number of mitotic figures and according to the WHO 2003 grading system (11), the tumor was classified as a borderline PT. IDC was also observed in a focal area of spindle cells (Fig. 3). The results of MaxVision ${ }^{\mathrm{TM}}$ 


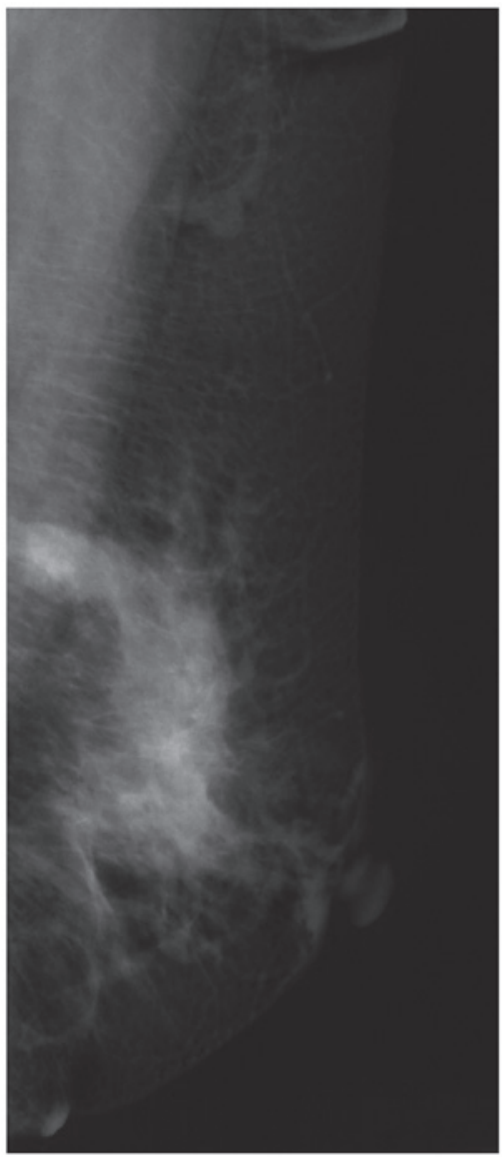

Figure 1. Mammography image revealing a high-density mass with an irregular margin and a diameter of $2.5 \mathrm{~cm}$ in the outer upper quadrant of the left breast. The mass was determined as grade 4, according to the Breast Imaging Reporting and Data System classification.

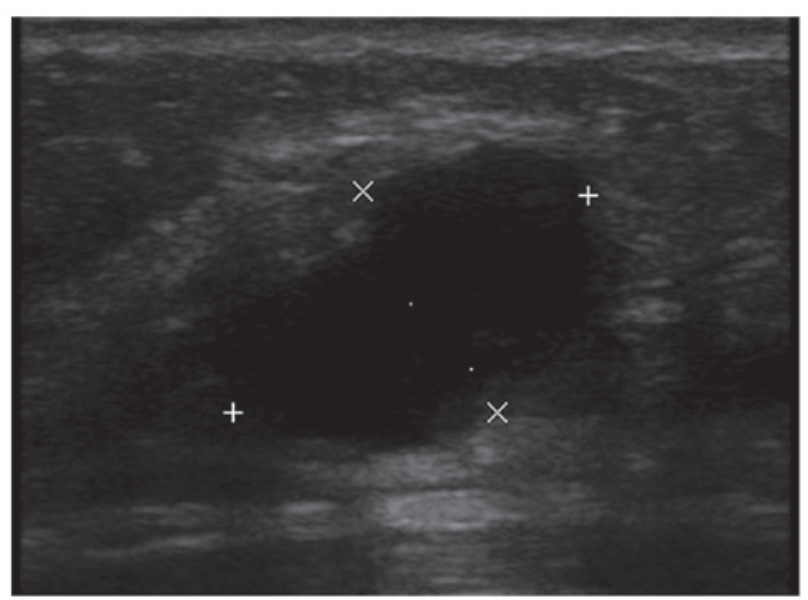

Figure 2. Sonographic examination revealing an irregular, partially ill-defined, hypoechoic mass with a diameter of $2.1 \mathrm{~cm}$. The mass was diagnosed as grade 4B, according to the Breast Imaging Reporting and Data System classification.

immunohistochemical staining (Fuzhou Maixin Biotechnology Development Co., Ltd., Fuzhou, China) of the IDC cells were as follows: Estrogen receptor negative; progesterone receptor negative; HER-2 negative; Ki-67 index, 30\%; cytokeratin (CK) 5/6 positive; vimentin positive; and pan-CK positive (Fig. 4).

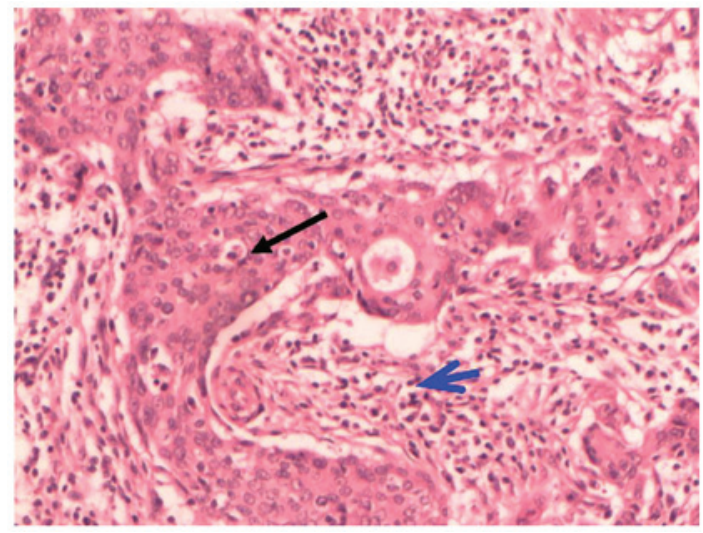

Figure 3. Invasive ductal carcinoma (black arrow) detected within a region of spindle cells (blue arrow) in the phyllodes tumor (hematoxylin and eosin stain; magnification, x100).

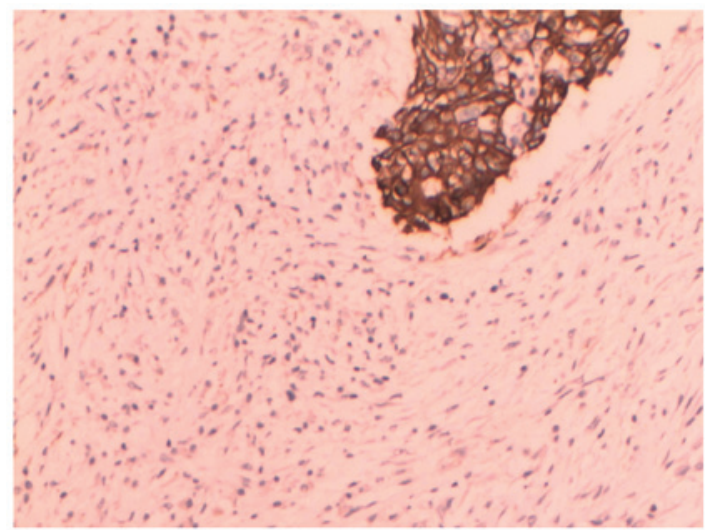

Figure 4. Immunohistochemical staining of the invasive ductal carcinoma, revealing positive pan-cytokeratin expression (stain, hematoxylin and eosin; magnification, $\mathrm{x} 100)$

Considering the diagnosis of IDC within a borderline PT, a simple mastectomy and sentinel lymph node biopsy (SLNB) were performed on July 10, 2012. Intraoperative frozen pathological analysis of 3 of the sentinel lymph nodes (SLNs) identified one micrometastasis. An axillary lymph node dissection and subsequent pathological examination did not reveal metastasis in any of the 18 nodes tested. The patient underwent six cycles of chemotherapy cycled every 21 days, consisting of $75 \mathrm{mg} / \mathrm{m}^{2}$ paclitaxel, $75 \mathrm{mg} / \mathrm{m}^{2}$ pirarubicin and $500 \mathrm{mg} / \mathrm{m}^{2}$ cyclophosphamide, all administered on day 1 . In addition, the patient underwent seven weeks of radiotherapy ( 25 cycles at 5,000 cGy; $200 \mathrm{cGy}$, each treatment). The tumor did not recur and no metastasis was observed during the first 23 months subsequent to treatment.

\section{Discussion}

PT may coexist with breast cancer in three situations. It may coexist in the bilateral breast, for example with IDC in one breast and a malignant PT in the other breast (12). PT has also been detected in the ipsilateral breast, such as IDC in the upper outer quadrant of the left breast and malignant PT in the lower outer quadrant of the left breast (13). Finally, PT may coexist with breast cancer in the same mass, as occurred in the current 


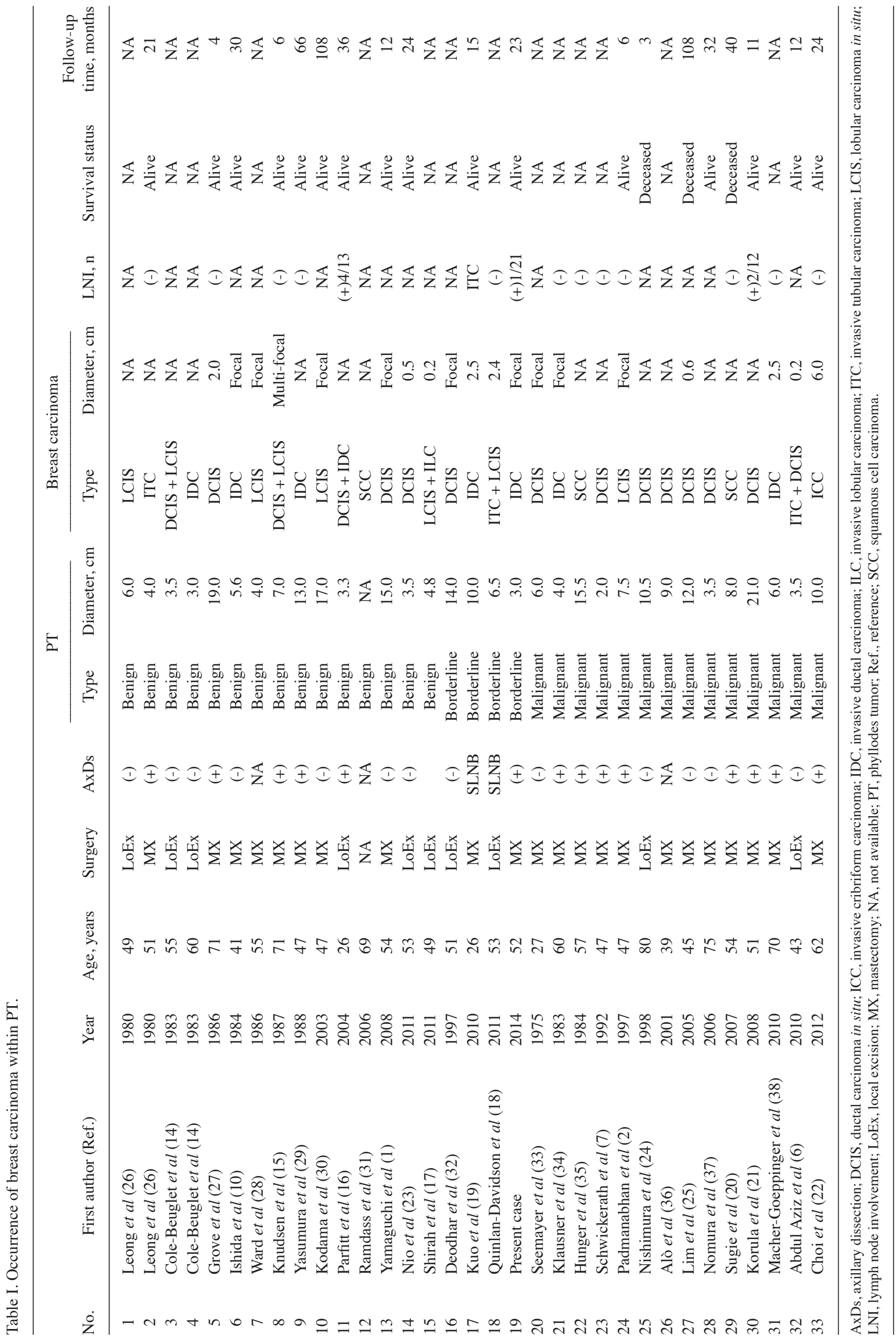


case. Breast carcinoma arising within PT is extremely rare. A literature search of the PubMed database (www.pubmed.com) was performed using the following search terms: 'breast cancer with phyllodes tumor' and 'coexistence of breast cancer and phyllodes tumor'. A total of 1,593 studies were retrieved. Using the following criteria, it was determined that $<40$ cases of breast carcinoma arising within PT have previously been reported in the literature (Table I) $(1,2,7,8,10,11,14-38)$. Inclusion criteria: i) published between 1974 and 2013; ii) English language; and iii) PT coexisting with breast cancer in the same tumor. Exclusion criteria: i) PT and breast cancer coexisting in the bilateral breast; ii) PT and breast cancer coexisting in the ipsilateral breast in different tumors; iii) no detailed pathological results; and iv) only the abstract available in English, full-text in a different language. The age of the patients with a coexistent breast carcinoma and PT ranged between 26 and 80 years, with a median age of 52 years. The reported breast carcinoma subtypes included in situ and invasive lobular and ductal (no specific type) carcinoma, invasive tubular carcinoma, squamous cell carcinoma and invasive cribriform carcinoma. Malignant epithelial elements were reported in all types of PT. Breast carcinoma was most commonly reported in malignant $(n=14)$ and benign $(n=15)$ PTs, but rarely in borderline PTs ( $n=4$, including the present case). Of the three cases of borderline PTs reported (excluding the present case), Kuo et al presented the case of a patient with a painless mass in the left breast, which had been present for 4 years. Following rapid growth of the tumor, the patient was diagnosed with invasive ductal carcinoma arising within a phyllodes tumor with isolated tumor cells identified in the sentinel lymph node (19). Mastectomy and sentinel lymph node biopsy were performed followed by hormonal therapy (goserelin acetate and tamoxifen), adjuvant chemotherapy (5-fluorouracil, epirubicin and cyclophosphamide) and reconstructive surgery. No tumor recurrence was reported during the 15 month follow-up period. Quinlan-Davidson et al (18) reported the case of a patient with a painless mass in the right breast that had been present for several years. Following two years of rapid growth of the mass the patient was diagnosed with borderline phyllodes tumor with an incidental invasive tubular carcinoma and lobular carcinoma in situ component. An excisional biopsy was performed and subsequently the patient underwent a re-excision for margin safety and a sentinel lymph node biopsy, which revealed that all three sentinel lymph nodes were negative for malignancy. In addition, Deodhar et al (32) reported a case of borderline phyllodes tumor with a ductal carcinoma in situ (DCIS) component. However, the outcome of the patient was not reported. Coexisting breast carcinoma within PTs more commonly demonstrated a ductal phenotype (IDC, n=7; DCIS, $\mathrm{n}=15$ ) compared with a lobular phenotype (ILC, $\mathrm{n}=1$; lobular carcinoma in situ, $\mathrm{n}=7$ ). A pure carcinoma in situ element was identified in 17 cases and was determined to be invasive in the other 16 cases. In addition, 6 cases were found to possess two types of malignant epithelial elements $(6,14-18)$. The PT size was not described in one case and the mean diameter of the tumor was $7.9 \pm 5.3 \mathrm{~cm}$. Yamaguchi et al (1) reported 7 cases of DCIS in PTs with a mean tumor size of $11.9 \mathrm{~cm}(15)$. Nio et al (23) reported that the mean diameter of breast carcinoma within PTs was $8.0 \mathrm{~cm}$ (14). The carcinoma size of the present case could not be measured.
In the previously reported literature, 10 cases were treated with local excision and 23 cases were treated with mastectomy. Of the 16 cases that received axillary surgery, three cases exhibited axillary lymph node metastasis and one possessed an isolated tumor cell in the SLN. As there is no standard adjuvant treatment strategy for this type of disease, a variety of systemic therapies were applied to the various cases. In total, 6 cases received chemotherapy with various regimens $(16,19-23), 5$ cases received radiotherapy $(13,16,18,20)$, and 4 cases received endocrine therapy, 3 of which received tamoxifen $(1,16,21)$ and 1 received tamoxifen and goserelin (19). Patient outcomes were described in 19 cases. The follow-up time was between 3 and 108 months. In total, 16 patients were alive at the end of last follow-up. Distance metastasis occurred in the lung in 2 cases at 3 and 32 months subsequent to surgery, respectively $(20,24)$. Similar to the current case, Kuo et al (19) and Parfitt et al (16) reported the combination of surgery, chemotherapy and radiotherapy for patients with lymph node metastasis who present breast carcinoma within PT. In addition, 3 cases succumbed to the disease, 3, 40 and 108 months subsequent to surgery, respectively $(20,24,25)$ (Table I).

In summary, the present study reports a rare case of IDC within a borderline PT. The imaging experiments performed lacked specificity. Instead, histology and immunohistochemistry are the golden standard for diagnosing this type of disease. The combination treatment of surgery, chemotherapy and radiotherapy was effective in the current case. Various types of breast carcinoma have been identified to coexist with PT in different masses; however, no standard therapeutic regimen has been established for the coexistence of PT and breast cancer in the same mass. The determination of an appropriate treatment strategy predominantly depends on the characteristics of the individual breast tumor, such as the hormone receptor status, HER-2 status and axillary lymph node metastasis status. Thus, future cases should undergo detailed analysis of tumor characteristics with reference to the molecular subtype and clinical pathological characteristics in order to select the optimal treatment strategy for breast cancer within phyllodes tumors.

\section{References}

1. Yamaguchi R, Tanaka M, Kishimoto Y, Ohkuma K, Ishida M and Kojiro M: Ductal carcinoma in situ arising in a benign phyllodes tumor: Report of a case. Surg Today 38: 42-45, 2008.

2. Padmanabhan V, Dahlstrom JE, Chong GC and Bennett G: Phyllodes tumor with lobular carcinoma in situ and liposarcomatous stroma. Pathology 29: 224-226, 1997.

3. Salvadori B, Cusumano F, Del Bo R, Delledonne V, Grassi M, Rovini D, Saccozzi R, Andreola S and Clemente C: Surgical treatment of phyllodes tumors of the breast. Cancer 63: 2532-2536, 1989

4. Bernstein L, Deapen D and Ross RK: The descriptive epidemiology of malignant cystosarcoma phyllodes tumors of the breast. Cancer 71: 3020-3024, 1993.

5. Lakhani SR, Ellis IO, Schnitt SJ, Tan PH and van de Vijver MJ (eds): WHO Classification of Tumours of the Breast. 4th edition. IARC Press, Lyon, 2012.

6. Abdul Aziz M, Sullivan F, Kerin MJ and Callagy G: Malignant phyllodes tumour with liposarcomatous differentiation, invasive tubular carcinoma and ductal and lobular carcinoma in situ: Case report and review of the literature. Patholog Res Int 2010: 501274, 2010.

7. Schwickerath J, Blessing MH and Wolff F: A rare clinical manifestation of a combination tumor of cystosarcoma phylloides malignum and an intraductal cancer. Geburtshilfe Frauenheilkd 52: 557-559, 1992 (In German). 
8. Stacher E, Boldt V, Leibl S, Halbwedl I, Popper HH, Ullmann R, Tavassoli FA and Moinfar F: Chromosomal aberrations as detected by array comparative genomic hybridization in early low-grade intraepithelial neoplasias of the breast. Histopathology 59: 549-555, 2011.

9. Arpino G, Bardou VJ, Clark GM and Elledge RM: Infiltrating lobular carcinoma of the breast: Tumor characteristics and clinical outcome. Breast Cancer Res 6: 149-156, 2004.

10. Ishida T, Izuo M and Kawai T: Breast carcinoma arising in cystosarcoma phyllodes: Report of a case with a review of the literature. Jpn J Clin Oncol 14: 99-106, 1984.

11. Tavassoli FA and Devilee P (eds): World Health Organization classification of tumours: Pathology and genetics of tumours of the breast and female genital organs. IARC Press, Lyon, pp99-103, 2003.

12. Merck B, Cansado Martínez P, Pérez Ramos M, Martinez Banaclocha N, Lacueva Gómez FJ and Calpena R: Infiltrating ductal carcinoma and synchronous malignant phyllodes tumour. Diagnostic and therapeutic approaches. Clin Transl Oncol 8: 830-832, 2006

13. Kefeli M, Yildiz L, Akpolat I, Balci P and Ozen N: The coexistence of invasive ductal carcinoma and malignant phyllodes tumor with liposarcomatous and chondrosarcomatous differentiation in the same breast in a post-osteosarcoma case. Pathol Res Pract 204: 919-923, 2008.

14. Cole-Beuglet C, Soriano R, Kurtz AB, Meyer JE, Kopans DB and Goldberg BB: Ultrasound, $x$-ray mammography and histopathology of cystosarcoma phylloides. Radiology 146: 481-486, 1983.

15. Knudsen PJ and Ostergaard J: Cystosarcoma phylloides with lobular and ductal carcinoma in situ. Arch Pathol Lab Med 111: 873-875, 1987

16. Parfitt JR, Armstrong C, O'Malley F, Ross J and Tuck AB In-situ and invasive carcinoma within a phyllodes tumor associated with lymph node metastases. World J Surg Oncol 2: 46, 2004.

17. Shirah GR, Lau SK, Jayaram L, Bouton ME, Patel PN and Komenaka IK: Invasive lobular carcinoma and lobular carcinoma in situ in a phyllodes tumor. Breast J 17: 307-309, 2011.

18. Quinlan-Davidson S, Hodgson N, Elavathil L and Shangguo T: Borderline phyllodes tumor with an incidental invasive tubular carcinoma and lobular carcinoma in situ component: A case report. J Breast Cancer 14: 237-240, 2011.

19. Kuo YJ, Ho DM, Tsai YF and Hsu CY: Invasive ductal carcinoma arising in phyllodes tumor with isolated tumor cells in sentinel lymph node. J Chin Med Assoc 73: 602-604, 2010

20. Sugie T, Takeuchi E, Kunishima F, Yotsumoto F and Kono Y: A case of ductal carcinoma with squamous differentiation in malignant phyllodes tumor. Breast Cancer 14: 327-332, 2007.

21. Korula A, Varghese J, Thomas M, Vyas F and Korula A: Malignant phyllodes tumour with intraductal and invasive carcinoma and lymph node metastasis. Singapore Med J 49: e318-e321, 2008.
22. Choi Y,Lee KY, Jang MH, Seol H, Kim SW and Park SY: Invasive cribriform carcinoma arising in malignant phyllodes tumor of breast: A case report. Korean J Pathol 46: 205-209, 2012.

23. Nio Y, Iguchi C, Tsuboi K and Maruyama R: Ductal carcinoma in situ arising within a benign phyllodes tumor: A case report with a review of the literature. Oncol Lett 2: 223-228, 2011.

24. Nishimura R, Hasebe T, Imoto S and Mukai K: Malignant phyllodes tumour with a noninvasive ductal carcinoma component. Virchows Arch 432: 89-93, 1998.

25. Lim SM and Tan PH: Ductal carcinoma in situ within phyllodes tumour: A rare occurrence. Pathology 37: 393-396, 2005.

26. Leong AS and Meredith DJ: Tubular carcinoma developing within a recurring cystosarcoma phyllodes of the breast. Cancer 46: 1863-1867, 1980.

27. Grove A and Deibjerg Kristensen L: Intraductal carcinoma within a phyllodes tumor of the breast: A case report. Tumori 72: 187-190, 1986.

28. Ward RM and Evans HL: Cystosarcoma phyllodes. A clinicopathologic study of 26 cases. Cancer 58: 2282-2289, 1986.

29. Yasumura T, Matsui S, Hamajima T, Nagashima K, Yamagishi $H$ Aikawa I, Oka T, Nakae T and Shimada N: Infiltrating ductal carcinoma developing within cystosarcoma phyllodes-a case report. Jpn J Surg 18: 326-329, 1988

30. Kodama T, Kameyama K, Mukai M, Sugiura H, Ikeda T and Okada Y: Invasive lobular carcinoma arising in phyllodes tumor of the breast. Virchows Arch 442: 614-616, 2003.

31. Ramdass MJ and Dindyal S: Phyllodes breast tumour showing invasive squamous-cell carcinoma with invasive ductal, clear-cell, secretory and squamous components. Lancet Oncol 7: 880, 2006.

32. Deodhar KK, Baraniya JB, Naresh KN, Shinde SR and Chinoy RF: Cancerization of phyllodes tumour. Histopathology 30: 98-99, 1997.

33. Seemayer TA, Tremblay G and Shibata H: The unique association of mammary stromal sarcoma with intraductal carcinoma. Cancer 36: 599-605, 1975.

34. Klausner JM, Lelcuk S, Ilia B, Inbar M, Hammer B, Skornik Y and Rozin RR: Breast carcinoma originating in cystosarcoma phyllodes. Clin Oncol 9: 71-74, 1983.

35. Hunger E, Turk R and Wurster K: Malignant cystosarcoma phylloides and squamous cell carcinoma of the breast. A rare tumor combination. Geburtshilfe Frauenheilkd 44: 640-642, 1984 (In German).

36. Alò PL, Andreano T, Monaco S, Sebastiani V, Eleuteri Serpieri D and Di Tondo U: Malignant phyllode tumor of the breast with features of intraductal carcinoma. Pathologica 93: 124-127, 2001 (In Italian).

37. Nomura M, Inoue Y, Fujita S, Sakao J, Hirota M, Souda S and Ohshima M: A case of noninvasive ductal carcinoma arising in malignant phyllodes tumor. Breast Cancer 13: 89-94, 2006.

38. Macher-Goeppinger S, Marme F, Goeppert B, Penzel R, Schirmacher P, Sinn HP and Aulmann S: Invasive ductal breast cancer within a malignant phyllodes tumor: Case report and assessment of clonality. Hum Pathol 41: 293-296, 2010. 